\title{
Vergleichender Blick auf Geschichte und Gegenwart von Präsidenten- und Ministeranklage
}

\begin{abstract}
Steinharth, Sebastian: Das Institut der Präsidenten- und Ministeranklage in rechtshistorischer und rechtsvergleichender Perspektive. Ursprünge, Erscheinungsformen und bleibende Sinnhaftigkeit von Gerichts- und Impeachmentverfahren zur Durchsetzung gubernativer Verantwortlichkeit (Hannoverisches Forum der Rechtswissenschaft, Band 36), Nomos Verlagsgesellschaft, Baden-Baden 2010, € 88,--
\end{abstract}

Die in Hannover entstandene Dissertation stellt die Frage nach der anhaltenden Bedeutung der Präsidenten- und Ministeranklage in den modernen präsidentiellen und parlamentarischen Regierungssystemen aus rechtshistorischer und - untrennbar verknüpft - rechtsvergleichender Perspektive. Obwohl diese Frage eingehender erst im dritten Teil (S. 204 ff.) behandelt wird und damit nur circa ein Drittel der gesamten Abhandlung ausmacht, wird das Terrain verfassungshistorisch-rechtsvergleichend in Teil 1 (spätmittelalterliche und frühneuzeitliche Vor- und Ausprägungen vornehmlich in England, S. 25 ff.) und Teil 2 (kontinentaleuropäische Aus- und Durchbildungen im Zeitalter des monarchischen Konstitutionalismus, S. 72 ff.) vorzüglich aufbereitet. Dabei werden nicht nur die Kontinuitätslinien gezogen, sondern es wird auch die gewandelte Bedeutung der Institute im frühneuzeitlichen England und monarchischen Konstitutionalismus herausgearbeitet. Die von Sebastian Steinharth eingangs konstatierte vorherrschende Negativbeurteilung (S. 19 m.w.N.) vermag der Rezensent - zumal aus der Sicht der ausländischen Literatur und der von Steinharth selbst angeführten Stimmen der deutschen Publikationen (S. 20, Fn. 4) nicht ganz zu teilen; sollte der Leser dennoch zur Gruppe der kritischen Beurteiler gehören, wird er im Laufe der Abhandlung durch das ausgebreitete rechtsnormative wie statistische Material (mit einem vorzüglichen Anhang über die Praxis der Präsidenten- und Ministeranklage, S. 313 f.) eines Besseren belehrt.

Steinharth behandelt entsprechend im ersten Teil die Verfahren zur Durchsetzung der gubernativen Verantwortlichkeit des Monarchen im Heiligen Römischen Reich, in England und Frankreich, wo sich in der frühen Neuzeit zunehmend der Gedanke der monarchischen Verantwortlichkeit im Rahmen absoluter Herrschaftsformen durchsetzte (S. 37 ff.), dem allerdings das Bestreben der Verantwortlichkeit der königlichen Räte korrespondiert, das in Form des englischen Impeachmentverfahrens für die konstitutionelle Ministerverantwortlichkeit Vorbildwirkung zeitigt (S. 70).

Teil 2 behandelt zunächst die konstitutionelle Ministerverantwortlichkeit vor dem rechtstheoretischen Hintergrund der Unverantwortlichkeit des Monarchen (John Locke, Montesquieu, Blackstone, Benjamin Constant, Robert von Mohl) und seiner Rechtfertigung in der Verfassungspraxis (S. 81 f.) im Sinne „gubernativer Verfassungs- und Gesetzesbindung“ (S. 86). Steinharth arbeitet zunächst die strafrechtliche Verantwortlichkeitsnatur (Frankreich, Belgien, Deutscher Bund und weitere Staaten) heraus, während die Abkehr von dieser Begründung im Sinne von Benjamin Constant hin zu einer konstitutionellen Kontrolle erst spät sich schrittweise entfaltet (S. 102, S. 169 f.). Institute wie das den monarchischen Konstitutionalismus prägende Gegenzeichnungsrecht der Minister (S. 114 f.) werden ebenso wie die Ausweitung des Verantwortlichkeitsgegenstands und -maßstabs thematisiert (S. 120 f.); die Ministerverantwortlichkeit zielte daher „vorrangig [auf] die Mitwirkung am Regierungshandeln des Monarchen in Gestalt der Schlechtberatung des Monarchen im 
Ministerrat oder aber die Gegenzeichnung seiner Anordnungen und Verfügungen“ (S. 146). Steinharth arbeitet die zwei Grundmodelle der Anklageverfahren in Form der Parlamentsgerichtsbarkeit (S. 172 f.) und der Hoch- beziehungsweise Staatsgerichtsbarkeit (S. 177 f.) beziehungsweise die Übertragung der Gerichtsbarkeit auf die höchsten ordentlichen Gerichte (S. 185 f.) heraus. Das Institut der bis zum heutigen Tag in vielfältigen Formen fortbestehenden Ministeranklage diente im monarchischen Konstitutionalismus dem konservativ-monarchischen Lager als Hemmschuh für eine weitere parlamentarische Kontrolle, dem liberaldemokratischen Lager als ein Vehikel, die parlamentarische Verantwortlichkeit zu verstärken; wie Steinharth zeigt, hatte sie im 19. Jahrhundert auch praktische Bedeutung vor allem in Skandinavien, aber auch in Frankreich, Griechenland und Italien.

In Teil 3 erörtert Steinharth die veränderte Bedeutung der Präsidenten- beziehungsweise Ministeranklage in parlamentarischen und präsidialen (oder gemischt-präsidialen) Systemen. Die Bedeutung des US-amerikanischen Impeachmentverfahrens tritt in den Fällen Andrew Johnson 1868, Richard Nixon 1974 oder Bill Clinton 1998 deutlich hervor (S. 206). Ob die in Art. 59 Satz 1 WRV verankerte Präsidentenanklage angesichts der Mehrheitsverhältnisse im Reichstag gegen Paul Hindenburg 1932 nicht nur politisch inopportun, sondern auch verfassungsrechtlich tragfähig gewesen wäre, bleibt eine offene Frage, die Steinharth natürlich nur streifen kann (S. 217). Er unterscheidet systematisch zwischen dem konkurrierenden Präsidentenanklageverfahren (Weimarer Verfassung, Deutschland, Österreich) und den exklusiven Präsidentenanklageverfahren (S. 226 f.), die jeweils ausführliche rechtsvergleichende Belege liefern. Der fortbestehende Sinngehalt der Präsidentenanklage wird zutreffend angenommen, auch wenn die Abgrenzung zum Organstreitverfahren, das alle Staaten mit ausgeprägter Verfassungsgerichtsbarkeit kennen, als vorrangiges Institut der Verfassungssicherung und Rechtsbindung auch des Präsidenten noch deutlicher hätte akzentuiert werden können; die Funktion der Präsidentenanklage als ein Damoklesschwert bleibt in der Regel bestehen. Schließlich widmet sich - wie schon eingangs bemerkt Steinharth ausdrücklich der Ministeranklage im modernen Verfassungsstaat (S. 243 f.), wo er wiederum zwischen den konkurrierenden Anklageverfahren (S. 245 f.; Weimarer Verfassung, Bundesrepublik Deutschland, Österreich und weitere Staaten) und den exklusiven Anklageverfahren (S. 274 f.: vorzügliche Darstellung insbesondere Frankreichs in der 3., 4. und 5. Republik, Belgiens und anderer Staaten) unterscheidet. Auch hier entfaltet sich die bleibende Bedeutung der Ministeranklage in der Möglichkeit der Amtsenthebung (S. 308), zumal die überwiegende Mehrzahl der Verfahren nicht das Misstrauensvotum gegen einen einzelnen Minister kennt, der Premier oder Regierungschef das Kabinettsmitglied aber jederzeit entlassen kann.

Insgesamt beeindruckt die Dissertation durch das reiche rechtshistorisch-vergleichende Material, das in mehr als 1.300 Fußnoten (!) auch ausführlich mit zum Teil zweisprachigen Texten belegt wird. Steinharth weist für den europäischen Raum und die USA 170 Verfahrensanläufe der Präsidenten- und Ministeranklagen nach, von denen circa die Hälfte nach erfolgreicher Parlamentsanklage das gerichtliche Verfahrensstadium erreichte; in 29 Fällen kam es zu einer Verurteilung und in 36 Fällen zu Freisprüchen (S. 311): Dies dürfte die Relevanz der untersuchten Institute nachdrücklich unterstreichen. 